\title{
Which Is the Fairest (Rent Division) of Them All?
}

\author{
YA'AKOV (KOBI) GAL, Ben-Gurion University, Israel \\ MOSHE MASH, Ben-Gurion University, Israel \\ ARIEL D. PROCACCIA, Carnegie Mellon University, USA \\ YAIR ZICK, Carnegie Mellon University, USA
}

"Mirror mirror on the wall, who is the fairest of them all?"

The Evil Queen

\begin{abstract}
What is a fair way to assign rooms to several housemates, and divide the rent between them? This is not just a theoretical question: many people have used the Spliddit website to obtain envy-free solutions to rent division instances. But envy freeness, in and of itself, is insufficient to guarantee outcomes that people view as intuitive and acceptable. We therefore focus on solutions that optimize a criterion of social justice, subject to the envy freeness constraint, in order to pinpoint the "fairest" solutions. We develop a general algorithmic framework that enables the computation of such solutions in polynomial time. We then study the relations between natural optimization objectives, and identify the maximin solution, which maximizes the minimum utility subject to envy freeness, as the most attractive. We demonstrate, in theory and using experiments on real data from Spliddit, that the maximin solution gives rise to significant gains in terms of our optimization objectives. Finally, a user study with Spliddit users as subjects demonstrates that people find the maximin solution to be significantly fairer than arbitrary envy-free solutions; this user study is unprecedented in that it asks people about their real-world rent division instances. Based on these results, the maximin solution has been deployed on Spliddit since April 2015.
\end{abstract}

CCS Concepts: •Applied computing $\rightarrow$ Economics; •Human-centered computing $\rightarrow$ User studies; - Theory of computation $\rightarrow$ Market equilibria;

Additional Key Words and Phrases: Computational fair division

\section{INTRODUCTION}

Many a reader may have personally experienced the rent division problem: several housemates move in together, and need to decide who gets which room, and at what price. The problem becomes interesting - and, more often than not, a source of frustration - when the rooms differ in quality. The challenge is then to achieve "rental harmony" [Su 1999] by assigning the rooms and dividing the rent fairly.

In more detail, suppose each player $i$ has value $v_{i j}$ for room $j$, such that each player's values for the rooms sum up to the total rent. The (quasilinear) utility of player $i$ for getting room $j$ at price $p_{j}$ is $v_{i j}-p_{j}$. A solution (i.e. an assignment of the rooms and division of the rent) is envy free [Foley 1967] if the utility of each player for getting

Authors' addresses: Y. Gal and M. Mash, Department of Information Systems Engineering, Ben-Gurion University, Israel; email: kobig@bgu.ac.il, mashm@post.bgu.ac.il. A. Procaccia and Y. Zick, Computer Science Department, Carnegie Mellon University, USA; email: \{arielpro, yairzick\}@cs . cmu .edu.

Permission to make digital or hard copies of all or part of this work for personal or classroom use is granted without fee provided that copies are not made or distributed for profit or commercial advantage and that copies bear this notice and the full citation on the first page. Copyrights for components of this work owned by others than ACM must be honored. Abstracting with credit is permitted. To copy otherwise, or republish, to post on servers or to redistribute to lists, requires prior specific permission and/or a fee. Request permissions from permissions@acm.org.

EC'16, July 24-28, 2016, Maastricht, The Netherlands.

ACM 978-1-4503-3936-0/16/07 ...\$15.00.

Copyright is held by the owner/author(s). Publication rights licensed to ACM.

http://dx.doi.org/10.1145/http://dx.doi.org/10.1145/2940716.2940724 
his room at its price is at least as high as getting any other room at the price of that room. More generally, one can think of this problem as allocating indivisible goods and splitting a sum of money - but we adopt the rent division terminology, which grounds the problem and justifies our assumptions.

Envy freeness is undoubtedly a compelling fairness notion. But what makes it truly powerful in the context of rent division is that an envy-free solution to a rent division problem always exists [Svensson 1983]. Even better, such a solution can be computed in polynomial time [Aragones 1995].

However, envy-freeness in and of itself is insufficient to guarantee satisfactory solutions. For example, consider an apartment with three rooms and total rent of $\$ 3$. Each player $i$ has value $\$ 3$ for room $i$, and value $\$ 0$ for the two other rooms. Furthermore, consider the solution that assigns room 1 to player 1 at $\$ 3$, and, for $i \in\{2,3\}$, gives room $i$ to player $i$ for free. This solution is envy free: players 2 and 3 are obviously overjoyed, while player 1 is indifferent between the three rooms. However, from an interpersonal perspective, this solution is not fair at all, as the distribution of prices between players is unequal. An intuitive alternative solution here would be to keep the same assignment of rooms, but equally split the rent between the different rooms - $\$ 1$ per room - thereby equalizing the utilities of the players.

The challenge, therefore, is to choose among many possible envy-free solutions. And, arguably, the most natural way to do this is to optimize a function of the utilities that meets desirable social criteria, subject to the envy-freeness constraint [Alkan et al. 1991]. In particular, if we were to maximize the minimum utility of any player subject to envy freeness, or if we were to minimize the maximum difference in utilities subject to envy freeness, we would obtain the aforementioned solution in the example. This focus on optimization in rent division motivates us to

... design polynomial-time algorithms for optimization under the envyfreeness constraint; understand the relationship between natural optimization objectives; and measure the theoretical and practical benefits of optimization in rent division.

\subsection{Real-World Connections and Implications: The Spliddit Service}

The above challenges are especially pertinent when put in the context of Spliddit (www.spliddit.org), a not-for-profit fair division website [Goldman and Procaccia 2014]. Spliddit offers "provably fair solutions" for the division of credit, indivisible goods, chores, fare - and, of course, rent. Since its launch in November 2014, Spliddit has attracted more than 60,000 users, who, in particular, have created 13,277 rent division instances (as of February 19, 2016).

Until April 2015, Spliddit's rent division application relied on the algorithm of Abdulkadiroğlu et al. [2004], which elicits the values of the players for the rooms, and computes an envy-free solution assuming quasi-linear utilities. While many users were satisfied with the results (based on their reported evaluations ${ }^{1}$ ), the algorithm does provide nonintuitive solutions in some cases. This prompted an investigation of alternative approaches, and ultimately led to the deployment of a new algorithm in April 2015 , based entirely on the results presented in this paper.

It is important to point out that Spliddit not only motivates our research questions, but also helps answer them. Indeed, while Spliddit's primary goals are making fair division methods accessible to people, and outreach, a secondary goal is the collection

\footnotetext{
${ }^{1}$ An example of one of many positive reviews: "This tool helped us a lot. We live in a flat populated by international, young people, so it's been almost a revolving door of roommates [...] With your method we were able to avoid any long discussions. Thank you."
} 
of an unprecedented dataset for fair division research [Goldman and Procaccia 2014]. This real-world dataset is exciting because, as noted by Herreiner and Puppe [2009], fair division is hard to study in the lab: researchers can tell subjects in the lab what their valuations are for different goods, but these values are not ecologically realistic, in that they do not represent subjects' actual preferences. To quote Herreiner and Puppe [2009], "the goods in the lab are not really distributed among participants, but serve as temporary substitutes for money." In contrast, Spliddit instances are ecologically valid, as they are posed by real people facing real division problems. Thus the Spliddit data enables studies at a realistic level and scale that was not possible before. Even better, we can ask Spliddit users to evaluate different solutions based on the actual instances they participated in. This is exactly what we do in this paper.

\subsection{Our Results}

We start, in Section 3, by constructing a general yet simple algorithmic framework for optimization under the envy-freeness constraint. Specifically, our algorithm maximizes the minimum of linear functions of the utilities, subject to envy freeness, in polynomial time. We do this by using the Second Welfare Theorem to argue that we can employ any welfare-maximizing assignment of players to rooms, and then solve a linear program to compute the optimal envy-free prices. ${ }^{2}$

Our main goal in Section 4 is to understand the relation between two solution concepts: the maximin solution [Alkan et al. 1991], which maximizes the minimum utility of any player subject to envy freeness; and the equitable solution, which minimizes disparity - the maximum difference in utilities - subject to envy freeness. (Our algorithm can compute either solution in polynomial time.) Our most significant result in this section is proving that the maximin solution is also equitable, but not every equitable solution is maximin.

Based on these results, we have implemented the polynomial-time algorithm of Section 3, with the maximin objective function. ${ }^{3}$ As noted above, it has been deployed on Spliddit since April 2015.

The remainder of the paper focuses on demonstrating that the foregoing approach is indeed effective, via theory and experiments. Here our contribution is twofold. First, we show - in Section 5 - that when values are drawn from a uniform Dirichlet distribution, and there are two or three players (the most common cases on Spliddit), the expected difference between the worst and best envy-free solutions in terms of disparity is significant. This means that, in theory, there is scope for significant improvement according to the equitability criterion. But do we also see an improvement in practice? We answer this question in the positive using Spliddit data. Indeed, we show that real-world instances give rise to significant differences, according to both the maximin and equitability objectives, between the maximin solution (which optimizes both objectives simultaneously) and an arbitrary envy-free solution (which does not attempt to optimize either objective).

Second, we report results from a user study, which has been a long time in the making. We contacted Spliddit users, and asked them to compare two solutions: the max-

${ }^{2}$ It is interesting to note that, even though the instances on Spliddit are small, computational tractability does play a key role, as there are many instances and computation incurs a cost (Spliddit uses Amazon Web Services to run all its algorithms).

${ }^{3}$ To be completely precise, the algorithm deployed on Spliddit first tries to maximize the minimum utility, subject to envy freeness as well as an additional constraint: prices must be non-negative. If an envy-free solution with non-negative prices does not exist [Brams and Kilgour 2001], it removes the non-negative price constraint (in which case a solution always exists). Most of our results go through even when prices are assumed to be non-negative. In any case, real-world instances where negative prices actually help are extremely rare, so throughout the paper prices are unconstrained. 
imin solution, and an arbitrary envy-free solution. Crucially, the two solutions were computed on each user's actual Spliddit instance (the values of other tenants were perturbed to preserve privacy). Subjects were asked to subjectively rate the solutions in terms of fairness to themselves, and fairness to others. The results show a significant advantage for the maximin solution in both questions, thereby demonstrating the added value of optimization and supporting the decision to use the maximin solution on Spliddit.

\subsection{Related Work}

The papers by Alkan et al. [1991] and Aragones [1995] are the most closely related to our work. Alkan et al. [1991] study the more general problem of allocating goods and dividing money. They start by proving the existence of envy-free solutions in this setting, but, like us, they ultimately employ criteria of justice in order to find the "best" envy-free solutions. They are especially interested in the maximin solution, which they call the value-Rawlsian solution; and the solution that maximizes the minimum amount of money allocated to any player, subject to envy freeness, which they call the money-Rawlsian solution. They show that the maximin solution is unique, as are a number of less attractive solutions (minimize the maximum utility, maximize the utility of one particular player). Finally, they show that these criteria imply solutions with a monotonicity property: if the amount of money is increased, the utility of all players is strictly higher (this property is moot in our setting). Alkan et al. [1991] do not provide algorithmic results.

Aragones [1995] designs a polynomial-time algorithm for computing the moneyRawlsian solution of Alkan et al. [1991]. Her combinatorial algorithm does not extend to other criteria. In contrast, our LP-based framework is significantly more general, and, in particular, allows us to compute the maximin solution (which we view as the most attractive) in polynomial time. Our algorithmic approach is also much simpler. It is worth noting that Klijn [2000] gives a different polynomial-time algorithm for computing envy-free solutions, without guaranteeing any additional properties (other than being extreme points of a certain polytope).

There are (at least) three marketlike mechanisms for computing solutions for the rent division problem assuming quasi-linear utilities, by Brams and Kilgour [2001], Haake et al. [2002], and Abdulkadiroğlu et al. [2004]. All three do not consider optimization criteria; in the case of the mechanism of Brams and Kilgour [2001], the solution may not be envy free. As mentioned above, the mechanism of Abdulkadiroğlu et al. [2004] was deployed on Spliddit until April 2015.

One fundamentally different approach to rent division that we would like to discuss in more detail is that of $\mathrm{Su}$ [1999]. He does not assume quasi-linear utilities; rather, his main assumption is that a player would always prefer getting a free room to getting another room at a positive price (the so-called miserly tenants assumption). Under this assumption, Su designs an algorithm that converges to an (approximately) envyfree solution, by iteratively querying players about their favorite room at given prices. While eschewing the quasi-linear utilities assumption is compelling, a (crucial, in our view) disadvantage of this approach is that preference elicitation is very cumbersome. Interestingly, Su's method was implemented by the New York Times. ${ }^{4}$

Relatively few papers explore fair allocations among people in lab settings, and there is inconclusive evidence about the types of solution criteria that are favored by people. Dupuis-Roy and Gosselin [2011] report that fair division algorithms were rated less desirable than imperfect allocations that did not employ any fairness criterion, while Schneider and Krämer [2004] find that subjects preferred envy-free solutions

\footnotetext{
${ }^{4} \mathrm{http}$ ///goo.gl/Xp3omV. This article also discusses the then-under-construction Spliddit.
} 
to a divide-and-choose method that does not guarantee envy-freeness. Herreiner and Puppe [2009, 2010] find that envy-freeness was a dominant factor in the allocations favored by subjects, but that it was a secondary criterion to Pareto optimality or inequality minimizing allocations. Kohler [2013] proposes an equilibrium strategy for repeated negotiation that incorporates fairness and envy concerns. In all of these papers, the studies were conducted in a controlled lab setting in which subjects' valuations over goods were imposed on the subjects, or the goods to be allocated were chosen by the experimenters themselves.

\section{THE MODEL}

We are interested in rent division problems involving a set of players $[n]=\{1, \ldots, n\}$, and a set of rooms $[n]$. Each player $i$ has a non-negative value $v_{i j} \in \mathbb{R}^{+}$for each room $j$. We assume without loss of generality that the total rent is 1 , and also assume (with loss of generality) that for all $i \in[n], \sum_{j=1}^{n} v_{i j}=1$. We can therefore represent an instance of the rent division problem as a right stochastic (rows sum to 1) matrix $V \in \mathbb{M}_{n \times n}\left(\mathbb{R}^{+}\right)$.

An assignment of the rooms is a permutation $\sigma:[n] \rightarrow[n]$, where $\sigma(i)$ is the room assigned to player $i$. The division of rent is represented through a vector of (possibly negative) prices $\mathbf{p} \in \mathbb{R}^{n}$ such that $\sum_{i=1}^{n} p_{i}=1 ; p_{j}$ is the price of room $j$.

Given a solution $(\sigma, \mathbf{p})$ for a rent division problem $V$, the quasi-linear utility of player $i$ is denoted $u_{i}(\sigma, \mathbf{p})=v_{i \sigma(i)}-p_{\sigma(i)}$. A solution is envy free $(\boldsymbol{E F})$ if the utility of each player for her room is at least as high as any other room. Formally, $(\sigma, \mathbf{p})$ is EF if and only if

$$
\forall i, j \in[n], v_{i \sigma(i)}-p_{\sigma(i)} \geq v_{i j}-p_{j} .
$$

\section{COMPUTATION OF OPTIMAL ENVY-FREE SOLUTIONS}

As noted above, it is possible to compute an envy-free solution to a given rent division problem in polynomial time [Aragones 1995]. We are interested in choosing among envy-free allocations by optimizing an objective function, subject to the envy-freeness constraint. Our goal in this section is to show that this can be done in polynomial time, when the objective function is the minimum of linear functions of the utilities.

THEOREM 3.1. Let $f_{1}, \ldots, f_{t}: \mathbb{R}^{n} \rightarrow \mathbb{R}$ be linear functions, where $t$ is polynomial in $n$. Given a rent division instance $V$, a solution $(\sigma, \mathbf{p})$ that maximizes the minimum of $f_{q}\left(u_{1}(\sigma, \mathbf{p}), \ldots, u_{n}(\sigma, \mathbf{p})\right)$ over all $q \in[t]$ subject to envy freeness can be computed in polynomial time.

Natural examples of objective functions of the form specified in the theorem are maximizing the minimum utility, and minimizing the maximum difference in utilities; we discuss these objectives in detail in Section 4 . The former objective can be directly captured by setting $t=n$, and $f_{i}\left(u_{1}(\sigma, \mathbf{p}), \ldots, u_{n}(\sigma, \mathbf{p})\right)=u_{i}(\sigma, \mathbf{p})$ for all $i \in[n]$. The latter criterion is also captured by setting $t=n^{2}$ and $f_{i j}\left(u_{1}(\sigma, \mathbf{p}), \ldots, u_{n}(\sigma, \mathbf{p})\right)=u_{i}(\sigma, \mathbf{p})-$ $u_{j}(\sigma, \mathbf{p})$. Indeed, $\min _{i, j \in[n]} f_{i j}\left(u_{1}(\sigma, \mathbf{p}), \ldots, u_{n}(\sigma, \mathbf{p})\right)=-\max _{i, j \in[n]}\left\{u_{i}(\sigma, \mathbf{p})-u_{j}(\sigma, \mathbf{p})\right\}$, so maximizing the minimum of these linear functions is equivalent to minimizing the maximum difference in utilities.

Our polynomial-time algorithm relies on a connection between envy-free rent division and the concept of Walrasian equilibrium. To understand this connection, imagine a more general setting where a set of buyers $[n]$ are interested in purchasing bundles of goods $G$; here, each buyer $i$ has a valuation function $v_{i}: 2^{G} \rightarrow \mathbb{R}$, assigning a value $v_{i}(S)$ to every bundle of goods. A Walrasian equilibrium is an allocation $\mathbf{A}=\left(A_{1}, \ldots, A_{n}\right)$ of the goods to buyers (where $A_{i} \subseteq G$ is the bundle given to buyer $i$ ), coupled with a price vector $\mathbf{p}$ that assigns a price to each good, such that each player receives the best 
bundle of goods that she can buy for the price $p$; formally:

$$
\forall i \in[n], S \subseteq G, v_{i}\left(A_{i}\right)-p\left(A_{i}\right) \geq v_{i}(S)-p(S) .
$$

We say that an allocation $\mathbf{A}$ is welfare-maximizing if it maximizes $\sum_{i=1}^{n} v_{i}\left(A_{i}\right)$. The following properties of Walrasian equilibria are well known; see, e.g., the book of MasColell et al. [1995, Chapter 16].

TheOREM 3.2 (1St Welfare TheOREM). If $(\mathbf{A}, \mathbf{p})$ is a Walrasian equilibrium, then $\mathbf{A}$ is a welfare-maximizing allocation.

THEOREM 3.3 (2ND Welfare THEOREM). If $(\mathbf{A}, \mathbf{p})$ is a Walrasian equilibrium, and $\mathbf{A}^{\prime}$ is a welfare-maximizing allocation, then $\left(\mathbf{A}^{\prime}, \mathbf{p}\right)$ is a Walrasian equilibrium as well. Furthermore, $v_{i}\left(A_{i}\right)-p\left(A_{i}\right)=v_{i}\left(A_{i}^{\prime}\right)-p\left(A_{i}^{\prime}\right)$ for all $i \in[n]$.

Now, an EF solution in the rent division setting is a Walrasian equilibrium in the setting where the goods are the rooms, and the valuation function of each player for a subset $S \subseteq[n]$ of rooms is given by $v_{i}(S)=\max _{j \in S} v_{i j}$ (these are unit demand valuations) - it is easily seen that Equation (1) coincides with Equation (2) in this case. This means that we can apply the welfare theorems to EF allocations. For example, we can immediately deduce a simple result of Svensson [1983]: any EF solution $(\sigma, \mathbf{p})$ is Pareto efficient, in the sense that there is no other solution $\left(\sigma^{\prime}, \mathbf{p}^{\prime}\right)$ such that $u_{i}\left(\sigma^{\prime}, \mathbf{p}^{\prime}\right) \geq u_{i}(\sigma, \mathbf{p})$ for all $i \in[n]$, with strict inequality for at least one $i \in[n]$. To see this, note that $\sigma$ is welfare-maximizing by Theorem 3.2, and the sum of prices is 1 under both $\mathbf{p}$ and $\mathbf{p}^{\prime}$.

We are now ready to present our polynomial-time algorithm for maximizing the minimum of linear functions $f_{1}, \ldots, f_{t}$ of the utilities, subject to EF; it is given as Algorithm 1.

\section{ALGORITHM 1:}

(1) Let $\sigma \in \operatorname{argmax}_{\pi}\left\{\sum_{i=1}^{n} v_{i \pi(i)}\right\}$ be a welfare-maximizing assignment

(2) Compute a price vector $\mathbf{p}$ by solving the linear program

$$
\begin{aligned}
& \max R
\end{aligned}
$$

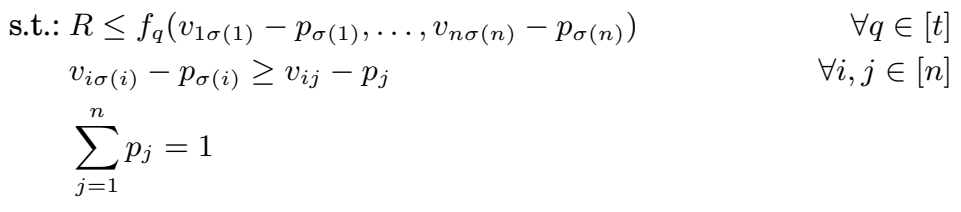

The algorithm starts by computing a welfare-maximizing assignment $\sigma$ of players to rooms; this can be done in polynomial time, as this task reduces to the maximum weight bipartite matching problem, with players on one side of the graph, rooms on the other, and a weight $v_{i j}$ on each edge $(i, j)$. It then solves (in polynomial time) a linear program, with variables $p_{1}, \ldots, p_{n}$, which computes optimal envy-free prices with respect to $\sigma$. The first constraint sets (in an optimal solution) the objective $R$ to the minimum of the linear functions $f_{q}(\cdot)$. Envy-freeness is enforced by the second constraint, and the third constraint guarantees that the prices sum to 1 .

However, it may not be immediately clear why starting from an arbitrary welfaremaximizing assignment allows us to compute the optimal solution subject to envyfreeness. This is formally established in the proof below. 
Proof of THEOREM 3.1. Let $\left(\sigma^{*}, \mathbf{p}^{*}\right)$ be the solution that maximizes the minimum of $f_{1}(\cdot), \ldots, f_{t}(\cdot)$ subject to EF. Furthermore, let $\sigma$ be the welfare-maximizing allocation computed in the first step of Algorithm 1. By Theorem 3.3, $\left(\sigma, \mathbf{p}^{*}\right)$ is such that $u_{i}\left(\sigma, \mathbf{p}^{*}\right)=u_{i}\left(\sigma^{*}, \mathbf{p}^{*}\right)$ for all $i \in N$. In particular, $\left(\sigma, \mathbf{p}^{*}\right)$ is $\mathbf{E F}$, and

$$
\min _{q \in[t]} f_{q}\left(u_{1}\left(\sigma, \mathbf{p}^{*}\right), \ldots, u_{n}\left(\sigma, \mathbf{p}^{*}\right)\right)=\min _{q \in[t]} f_{q}\left(u_{1}\left(\sigma^{*}, \mathbf{p}^{*}\right), \ldots, u_{n}\left(\sigma^{*}, \mathbf{p}^{*}\right)\right) .
$$

Because $\mathbf{p}^{*}$ is a feasible solution to the linear program, we have that its optimal solution $\mathrm{p}$ satisfies

$$
\min _{q \in[t]} f_{q}\left(u_{1}(\sigma, \mathbf{p}), \ldots, u_{n}(\sigma, \mathbf{p})\right) \geq \min _{q \in[t]} f_{q}\left(u_{1}\left(\sigma, \mathbf{p}^{*}\right), \ldots, u_{n}\left(\sigma, \mathbf{p}^{*}\right)\right) .
$$

Putting Equations (3) and (4) together, we see that we must have equality in (4), and that $(\sigma, \mathbf{p})$ is an optimal envy-free solution.

\section{RELATIONS BETWEEN THE FAIREST SOLUTIONS}

Algorithm 1 allows us to maximize the minimum of linear functions of the utilities, subject to EF, in polynomial time. With the potential computational barrier out of the way, we would like to understand which optimization objective to use. Specifically, we focus on two natural optimization objectives, and evaluate their properties.

We refer to the first objective as equitability. Let $E F(V)$ be the set of all EF outcomes for $V$. Given an outcome $(\sigma, \mathbf{p}) \in E F(V)$, we define $D(\sigma, \mathbf{p})$ as the difference between the utilities of the happiest player and the worst off player under the outcome $(\sigma, \mathbf{p})$, that is,

$$
D(\sigma, \mathbf{p})=\max _{i, j \in N}\left\{u_{i}(\sigma, \mathbf{p})-u_{j}(\sigma, \mathbf{p})\right\} .
$$

In more general terms, the function $D$ measures the social disparity under the outcome $(\sigma, \mathbf{p})$; we would like to minimize this quantity. An outcome $\left(\sigma^{*}, \mathbf{p}^{*}\right)$ is called equitable if it minimizes $D$ over $E F(V)$, i.e.,

$$
\left(\sigma^{*}, \mathbf{p}^{*}\right) \in \arg \min \{D(\sigma, \mathbf{p}) \mid(\sigma, \mathbf{p}) \in E F(V)\} .
$$

Herreiner and Puppe [2009] demonstrate via experiments with human subjects that equitability is of great importance in determining whether an allocation is perceived to be fair by people.

Alternatively, instead of minimizing social disparity, one might be interested in maximizing the utility of the worst off player. More formally, given an $\mathrm{EF}$ solution $(\sigma, \mathbf{p})$, we let $U(\sigma, \mathbf{p})=\min _{i \in N} u_{i}(\sigma, \mathbf{p})$; if

$$
\left(\sigma^{*}, \mathbf{p}^{*}\right) \in \arg \max \{U(\sigma, \mathbf{p}) \mid(\sigma, \mathbf{p}) \in E F(V)\}
$$

then we say that $\left(\sigma^{*}, \mathbf{p}^{*}\right)$ is a maximin solution.

Alkan et al. [1991] argue that the maximin solution - which they call the valueRawlsian solution - is compelling on philosophical grounds. Mathematically, they demonstrate that the maximin solution is associated with a unique vector of utilities, making this solution even more appealing.

The fact that equitable and maximin allocations are constrained to be $\mathrm{EF}$ again allows us to employ the Second Welfare Theorem (Theorem 3.3) to great effect. Indeed, if $\left(\sigma^{*}, \mathbf{p}^{*}\right)$ is equitable (resp., maximin), and $\sigma^{\prime}$ is a welfare-maximizing assignment, then $\left(\sigma^{\prime}, \mathbf{p}^{*}\right)$ is equitable (resp., maximin). Therefore, hereinafter we assume without loss of generality that the identity assignment $\sigma(i)=i$ is welfare maximizing, and simply use $D(\mathbf{p})$ or $U(\mathbf{p})$ to refer to these measures under the identity assignment. In particular, we can talk about equitable or maximin vectors of prices with respect to the identity assignment. 
At first glance, the equitability and maximin criteria seem equally appealing. Which one leads to fairer solutions? The next theorem shows that we do not have to choose the maximin solution is equitable.

THEOREM 4.1. If $\mathbf{p}^{*}$ is a maximin vector of prices, then it is also equitable.

PROoF. Given an EF vector of prices p, it will be useful to think of the following graph $\Gamma(\mathbf{p})=([n], E)$; the nodes in $\Gamma(\mathbf{p})$ are the players, and there is a directed edge from $i$ to $j$ if $i$ weakly envies $j$ - that is, $v_{i i}-p_{i}=v_{i j}-p_{j}$ (recall that we are assuming the identity assignment). We say that $i$ is poor if $i$ has minimum utility under $\mathbf{p}$; similarly, a player $i$ is called rich if $i$ has maximum utility under $\mathbf{p}$.

We first claim that if $\mathbf{p}^{*}$ is a maximin rent division, then every non-poor player $i$ has a path to a poor player in $\Gamma\left(\mathbf{p}^{*}\right)$. Indeed, assume for contradiction that this is not the case, and let $T^{*}$ be the set of all players that have no path to poor players under $\Gamma\left(\mathbf{p}^{*}\right)$;

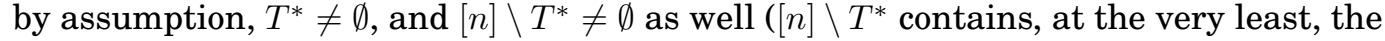
poor players, who have a path of length 0 to themselves). Let us observe the vector of prices q defined as follows:

$$
q_{i}= \begin{cases}p_{i}+\varepsilon & i \in T^{*} \\ p_{i}-\frac{\left|T^{*}\right| \varepsilon}{n-\left|T^{*}\right|} & i \in[n] \backslash T^{*}\end{cases}
$$

Here, $\varepsilon$ is a very small constant, which is in particular smaller than $\min \left\{u_{i}\left(i d, \mathbf{p}^{*}\right)-\right.$ $\left.u_{j}\left(i d, \mathbf{p}^{*}\right) \mid u_{i}\left(i d, \mathbf{p}^{*}\right)>u_{j}\left(i d, \mathbf{p}^{*}\right)\right\}$. Let $i, j \in[n]$ such that $i$ weakly envies $j$ under $\mathbf{p}^{*}$. If $i, j \in T^{*}$ or $i, j \in[n] \backslash T^{*}$ then $i$ still weakly envies $j$ : their prices changed by the same amount. If $i$ belongs to $T^{*}$ then $j$ cannot belong to $[n] \backslash T^{*}$, otherwise there would be a path from $i$ to some poor player, a contradiction to the definition of $T^{*}$. If $i$ belongs to $[n] \backslash T^{*}$ and $j$ belongs to $T^{*}$ then $i$ enjoyed a decrease in rent, whereas $j$ suffered an increase, so $i$ does not envy $j$ under q. We conclude that q is envy free, by our choice of $\varepsilon$. However, because $[n] \backslash T^{*}$ includes all poor players, the minimum utility under $\mathbf{q}$ is strictly higher than under $\mathbf{p}^{*}$, a contradiction to $\mathbf{p}^{*}$ being a maximin EF rent division.

Next, let $\mathbf{q}^{*}$ be an equitable EF price vector. Suppose for contradiction that $D\left(\mathbf{p}^{*}\right)>$ $D\left(\mathbf{q}^{*}\right)$. If all players have the same utility under $\mathbf{p}^{*}$ then $D\left(\mathbf{p}^{*}\right)=0 \leq D\left(\mathbf{q}^{*}\right)$ which is impossible. Hence there must be some rich players that are not poor under $\mathbf{p}^{*}$.

We write $\varepsilon=U\left(\mathbf{p}^{*}\right)-U\left(\mathbf{q}^{*}\right)$; since $\mathbf{p}^{*}$ is a maximin EF rent division, $\varepsilon \geq 0$. This means that every poor player under $\mathbf{p}^{*}$ could have had their utility decreased by at most $\varepsilon$. In other words, if $i$ is a poor player under $\mathbf{p}^{*}$, then $q_{i}^{*}-p_{i}^{*} \leq \varepsilon$. Moreover, since $D\left(\mathbf{p}^{*}\right)>D\left(\mathbf{q}^{*}\right)$ by assumption, it must be the case that the rich players under $\mathbf{p}^{*}$ had their utility decreased by strictly more than $\varepsilon$; this is because if $i$ is a rich player then

$$
u_{i}\left(i d, \mathbf{p}^{*}\right)-\min _{j \in[n]} u_{j}\left(i d, \mathbf{p}^{*}\right)>\max _{j \in[n]} u_{j}\left(i d, \mathbf{q}^{*}\right)-\min _{j \in[n]} u_{j}\left(i d, \mathbf{q}^{*}\right),
$$

and therefore $u_{i}\left(i d, \mathbf{p}^{*}\right)>\max _{j \in[n]} u_{j}\left(i d, \mathbf{q}^{*}\right)+\varepsilon$, which implies that $u_{i}\left(i d, \mathbf{p}^{*}\right)>$ $u_{i}\left(i d, \mathbf{q}^{*}\right)+\varepsilon$, or, equivalently, $q_{i}^{*}-p_{i}^{*}>\varepsilon$.

We know that there is a path from at least one rich player (who is not poor) to at least one poor player in $\Gamma\left(\mathbf{p}^{*}\right)$. In particular, there must be an edge $(i, j)$ on that path such that $q_{i}^{*}-p_{i}^{*}>q_{j}^{*}-p_{j}^{*}$. By the definition of $\Gamma\left(\mathbf{p}^{*}\right), v_{i i}-p_{i}^{*}=v_{i j}-p_{j}^{*}$. It follows that

$$
v_{i i}-q_{i}^{*}=v_{i i}-p_{i}^{*}+\left(p_{i}^{*}-q_{i}^{*}\right)<v_{i j}-p_{j}^{*}+\left(p_{j}^{*}-q_{j}^{*}\right)=v_{i j}-q_{j}^{*},
$$

contradicting the envy freeness of $\mathbf{q}^{*}$.

In contrast, an equitable solution may not be maximin, as the following example shows. 
Example 4.2 (An equitable solution that is not maximin). This example is particularly appealing, as it is a real-world instance submitted by Spliddit users.

$$
\left(\begin{array}{ccc}
2227 & 708 & 0 \\
258 & 1378 & 1299 \\
1000 & 1000 & 935
\end{array}\right)
$$

Note that the total rent is $\$ 2935$. The optimal room assignment gives room $i$ to player $i$; the maximin rent division is $\mathbf{p}^{*}=\left(1813 \frac{1}{3}, 600 \frac{1}{3}, 521 \frac{1}{3}\right)$, with a utility vector of $u_{1}\left(i d, \mathbf{p}^{*}\right)=413 \frac{2}{3}, u_{2}\left(i d, \mathbf{p}^{*}\right)=777 \frac{2}{3}, u_{3}\left(i d, \mathbf{p}^{*}\right)=413 \frac{2}{3}$. We have $D\left(\mathbf{p}^{*}\right)=777 \frac{2}{3}-$ $413 \frac{2}{3}=364$, and by Theorem 4.1 any solution that has the same disparity is equitable. However, the price vector $\mathbf{p}^{\prime}=\left(1570 \frac{2}{3}, 721 \frac{2}{3}, 642 \frac{2}{3}\right)$ is an EF rent division resulting in $u_{1}\left(i d, \mathbf{p}^{\prime}\right)=656 \frac{1}{3}, u_{2}\left(i d, \mathbf{p}^{\prime}\right)=656 \frac{1}{3}, u_{3}\left(i d, \mathbf{p}^{\prime}\right)=292 \frac{1}{3}$, and $D\left(\mathbf{p}^{\prime}\right)=656 \frac{1}{3}-292 \frac{1}{3}=364$ as well, that is, it is equitable, but the minimum utility is (much) smaller than that under $\mathbf{p}^{*}$.

Let us now discuss a third optimization objective, the money-Rawlsian solution, which is mentioned by Alkan et al. [1991], and implemented in polynomial time by Aragones [1995]. The latter author describes the following procedure for finding EF solutions. Begin by finding a welfare-maximizing assignment of rooms (again, assume without loss of generality that room $i$ goes to player $i$ ); next, find a vector $\mathbf{q}^{*} \in \mathbb{R}_{+}^{n}$ of non-negative values such that $v_{i i}+q_{i}^{*} \geq v_{i j}+q_{j}^{*}$ and $Q^{*}=\sum_{i=1}^{n} q_{i}^{*}$ is minimized. That is, each player $i$ pays a value of $-q_{i}^{*}$. Next, increase the prices of all players by a quantity $\alpha$ such that $n \alpha-Q^{*}=1$, i.e. the vector $(\alpha, \ldots, \alpha)-\mathbf{q}^{*}$ is a valid price vector.

While the money-Rawlsian solution is interesting, it may be "maximally unfair" in terms of disparity, as the following example shows.

Example 4.3 (The money-Rawlsian solution may maximize disparity). We analyze the following rent division instance:

$$
V=\left(\begin{array}{cc}
1 & 0 \\
\frac{1}{2} & \frac{1}{2}
\end{array}\right)
$$

The welfare-maximizing assignment allocates room $i$ to player $i$, and $\mathbf{q}^{*}=(0, \ldots, 0)$. A uniform increase in rent will ensue, resulting in the price vector $(1 / 2,1 / 2)$ and the utility vector $(1 / 2,0)$. Crucially, the money-Rawlsian price vector maximizes disparity among all EF solutions. Note that the maximin price vector is $(3 / 4,1 / 4)$, which, of course, minimizes disparity.

To conclude, so far we know that the maximin solution, the equitable solution, and the money-Rawlsian solution can be computed in polynomial time. Moreover, Theorem 4.1 shows that the maximin solution, which by definition maximizes the minimum utility, also minimizes disparity (among all EF solutions) - so it is a refinement of the equitable solution. In stark contrast, the money-Rawlsian solution may maximize disparity (among all EF solutions). We therefore view the maximin solution as the clear choice, and focus on analyzing its effectiveness hereinafter.

\section{ON THE IMPORTANCE OF BEING EQUITABLE}

Our goal in this section is to understand how much better the maximin solution is, in terms of the maximin and disparity objectives, compared to suboptimal solutions on average. In Section 5.1 we show that the expected gain in terms of reducing disparity is significant in a formal probabilistic model. For this theoretical analysis, we focus on the cases of two and three players, which are the most common on Spliddit. We also 
focus on the equitability criterion, but the same ideas can be applied to the maximin criterion. In Section 5.2 we conduct an empirical analysis, showing significant gains in both of our primary objective functions on real data from Spliddit.

\subsection{The Benefit Is Significant in Theory}

Given an $n$ player rent division problem, let $D^{+}(V)=\max _{\mathbf{p} \in E F(V)} D(\mathbf{p})$, and $D^{*}(V)=$ $\min _{\mathbf{p} \in E F(V)} D(\mathbf{p})$; that is, $D^{+}(V)$ is the highest utility difference between the best and worst-off players under the disparity maximizing EF solution, whereas $D^{*}(V)$ is the difference between the best and worst-off players under the equitable solution discussed earlier. In order to establish the potential impact of minimizing disparity, we would like to show that instances where the difference $D^{+}(V)-D^{*}(V)$ is significant are a common occurrence.

More formally, we are interested in computing $\mathbb{E}_{V \sim \mu}\left[D^{+}(V)-D^{*}(V)\right]$ for rent division instances sampled from some distribution $\mu$ over $n$ player rent division instances. In our theoretical results below, we use the uniform Dirichlet distribution over each player's values for the $n$ rooms. In more detail, each $i \in[n]$ chooses a valuation profile uniformly at random from the $n-1$ dimensional simplex. Such uniform distributions can be generated as follows: let $X_{1}, \ldots, X_{n-1} \sim \mathcal{U}[0,1]$ be independent uniform random variables on $[0,1]$, and let $X_{(1)} \leq \cdots \leq X_{(n-1)}$ be the variables sorted according to their order statistics; then the vector $\mathbf{v}=\left(X_{1}, X_{(2)}-X_{(1)}, \ldots, X_{(n-1)}-X_{(n-2)}, 1-X_{(n-1)}\right)$ corresponds to a sample from the uniform Dirichlet distribution.

The two player case. Let us start with the setting where there are only two players. In that case, a valuation matrix can be written as

$$
\left(\begin{array}{ll}
v_{1} & 1-v_{1} \\
v_{2} & 1-v_{2}
\end{array}\right)
$$

where $v_{1}, v_{2} \in[0,1]$. To draw a random instance, we simply need to draw $v_{1}, v_{2} \sim \mathcal{U}[0,1]$. We use two lemmas to exactly calculate $\mathbb{E}_{V \sim \mu}\left[D^{+}(V)-D^{*}(V)\right]$.

Lemma 5.1. Let $n=2$. Then there exists an EF price vector $\mathbf{p}^{-}$such that $D\left(\mathbf{p}^{-}\right)=0$.

PROOF. We again assume that the identity assignment is welfare maximizing. Given a price vector $\left(p_{1}, p_{2}\right)$, the player utilities are $v_{1}-p_{1}$ and $1-v_{2}-p_{2}$; setting $p_{2}=1-p_{1}$ and solving for $v_{1}-p_{1}=1-v_{2}-p_{2}$ yields $p_{1}=\frac{v_{1}+v_{2}}{2}$.

It remains to make sure that this solution is indeed envy free. Under this price vector we have that player 1's utility from room 1 is $\frac{v_{1}-v_{2}}{2}$, and her utility from room 2 is $1-v_{1}-\left(1-\frac{v_{1}+v_{2}}{2}\right)=\frac{v_{2}-v_{1}}{2}$. Now, if player 1 envies player 2 , then $v_{1}<v_{2}$, in which case allocating room $i$ to player $i$ is not a welfare-maximizing assignment. To see this, note that

$$
v_{1}+1-v_{2} \geq v_{2}+1-v_{1} \Longleftrightarrow v_{1} \geq v_{2} .
$$

Thus, under this price vector, player 1 does not envy player 2 . Now, player 2's utility from room 2 is $\frac{v_{1}-v_{2}}{2}$, and her utility from room 1 is $v_{2}-\frac{v_{1}+v_{2}}{2}=\frac{v_{2}-v_{1}}{2}$, which is not more than her utility from her own room as previously argued.

To conclude, $\mathbf{p}^{-}=\left(\frac{v_{1}+v_{2}}{2}, 1-\frac{v_{1}+v_{2}}{2}\right)$ is an EF price vector for which $D\left(\mathbf{p}^{-}\right)=0$.

LEMMA 5.2. Given a 2 player rent division instance

$$
V=\left(\begin{array}{ll}
v_{1} & 1-v_{1} \\
v_{2} & 1-v_{2}
\end{array}\right)
$$

it holds that $D^{+}(V)=\left|v_{1}-v_{2}\right|$. 
PROOF. Suppose that $v_{1} \geq v_{2}$ (the case of $v_{1}<v_{2}$ is handled similarly). In this case, we can assume that room $i$ is assigned to player $i$. A price vector $\left(p_{1}, p_{2}\right)$ is $\mathrm{EF}$ if and only if $v_{2} \leq p_{1} \leq v_{1}$. Since $D(\mathbf{p})$ is the maximum of linear functions, its maxima occur on vertices of the polyhedron of EF rent divisions. Thus, the maximum difference in player utilities must occur when $p_{1}$ equals either $v_{1}$ or $v_{2}$. Note that $u_{1}\left(v_{1}, 1-v_{1}\right)=$ $0, u_{2}\left(v_{1}, 1-v_{1}\right)=v_{1}-v_{2}$, and $u_{1}\left(v_{2}, 1-v_{2}\right)=v_{1}-v_{2}, u_{2}\left(v_{2}, 1-v_{2}\right)=0$. Thus, in either case $D^{+}(V)=v_{1}-v_{2}$.

Therefore, in order to estimate $\mathbb{E}\left[D^{+}(V)-D^{*}(V)\right]$ in the two player case, we simply need to calculate $\mathbb{E}\left[\left|v_{1}-v_{2}\right|\right]$, which is clearly $1 / 3$. We include the proof for completeness.

THEOREM 5.3. Let

$$
V=\left(\begin{array}{ll}
v_{1} & 1-v_{1} \\
v_{2} & 1-v_{2}
\end{array}\right)
$$

be a 2 player rent division instance, where $v_{1}, v_{2} \sim \mathcal{U}(0,1)$; then $\mathbb{E}\left[D^{+}(V)-D^{*}(V)\right]=\frac{1}{3}$.

Proof. According to Lemmas 5.1 and 5.2, $D^{+}(V)=\left|v_{1}-v_{2}\right|$ and $D^{*}(V)=0$. Thus we simply need to calculate

$$
\mathbb{E}\left[D^{+}(V)\right]=\mathbb{E}\left[\left|v_{1}-v_{2}\right|\right]=\frac{1}{2} \mathbb{E}\left[v_{1}-v_{2} \mid v_{1} \geq v_{2}\right]+\frac{1}{2} \mathbb{E}\left[v_{2}-v_{1} \mid v_{2} \geq v_{1}\right] .
$$

Since both $v_{1}$ and $v_{2}$ are i.i.d., we have that $\mathbb{E}\left[v_{1}-v_{2} \mid v_{1} \geq v_{2}\right]=\mathbb{E}\left[v_{2}-v_{1} \mid v_{2} \geq v_{1}\right]$ (note that this does not use the fact that $v_{1}, v_{2}$ are uniform on $[0,1]$ ). Finally, we have that

$$
\begin{aligned}
\mathbb{E}\left[v_{1}-v_{2} \mid v_{1}>v_{2}\right] & =\int_{0}^{1} \int_{0}^{v_{1}}\left(v_{1}-v_{2}\right) d v_{2} d v_{1}=\frac{1}{2}-\int_{0}^{1} \int_{0}^{v_{1}} v_{2} d v_{2} d v_{1} \\
& =\frac{1}{2}-\frac{1}{2} \int_{0}^{1} v_{1}^{2} d v_{1}=\frac{1}{2}-\frac{1}{2} \cdot \frac{1}{3}=\frac{1}{3}
\end{aligned}
$$

Three Players, and Beyond. We now proceed to tackle the 3 player rent division case, in the foregoing regime. While our results for this case are not nearly as tight as for the case of two players, we provide an in-depth analysis of an interesting class of three player rent division instances. This class includes instances where all players mostly agree on the value of one room, but disagree on the values of the other two.

More formally, let $\mathcal{C}^{*}(\varepsilon)$ be the class of 3 player rent division instances that satisfy the following property: there exists some room $j$ for which $\left|v_{i j}-v_{k j}\right| \leq \varepsilon$ for all $i, k \in[n]$, but $\left|v_{i \ell}-v_{k \ell}\right| \geq 2 \varepsilon$ for all $\ell \in[n] \backslash\{j\}$ and all $i \in[n], k \in[n] \backslash\{i\}$. The next lemma whose proof is given in the full version of this paper ${ }^{5}$ - shows that if a rent division instance $V$ is in $\mathcal{C}^{*}(\varepsilon)$, then $V$ allows for an extremely equitable EF solution, where each player has utility of nearly $\left(\sum_{i} v_{i i}-1\right) / 3$; however, it also admits an EF solution where one of the players has utility 0 - the worst possible outcome.

LEMMA 5.4. If $V \in \mathcal{C}^{*}(\varepsilon)$, then there exist EF price vectors $\mathbf{p}^{+}$and $\mathbf{p}^{-}$such that $U\left(\mathbf{p}^{-}\right) \geq \frac{\sum_{i} v_{i i}-1}{3}-\frac{2}{3} \varepsilon$, but $U\left(\mathbf{p}^{+}\right)=0$. Moreover, $D^{*}(V) \leq \varepsilon$, but $D^{+}(V) \geq 2 \varepsilon$.

By proving a lower bound on the probability that rent division instances belong to $\mathcal{C}^{*}(\varepsilon)$, we can establish the following theorem, whose proof appears in the full version of this paper.

\footnotetext{
${ }^{5}$ Available from: http://procaccia.info/research
} 
THEOREM 5.5. Let $V$ be a 3 player rent division instance drawn from the uniform Dirichlet distribution; then for any $\varepsilon<1 / 5$,

$$
\operatorname{Pr}\left[D^{+}(V)-D^{*}(V) \geq \varepsilon\right] \geq 18 \varepsilon^{2}-312 \varepsilon^{3}+1902 \varepsilon^{4}-4560 \varepsilon^{5}+\frac{15189}{5} \varepsilon^{6} .
$$

For example, Theorem 5.5 implies that with probability at least $0.02, D^{+}(V)-D^{*}(V) \geq$ 0.07 (which is $7 \%$ of the total rent). With smaller probability of roughly 0.0025 , the difference is huge - almost $20 \%$ of the total rent.

In contrast, it is intuitive that as $n$ grows, we cannot expect the difference in disparity to remain bounded away from zero. The reason is that for any fixed $\varepsilon>0$, it is likely that all players agree on the values of all rooms up to $\varepsilon$; that is, $\left|v_{i k}-v_{j k}\right|<\varepsilon$ for all $i, j, k \in[n]$ with high probability. This property guarantees that near equitability holds for all EF rent divisions. Indeed, when all players agree on all values up to $\varepsilon$, choosing different EF price vectors causes little difference in players' utilities; in a sense, there is very little "wiggle room" due to players' utility vectors being so similar to one another. Formally:

LEMMA 5.6. If $V$ is such that $\left|v_{i k}-v_{j k}\right|<\varepsilon$ for all $i, j, k \in[n]$, then $D(\mathbf{p}) \leq \varepsilon$ for all EF payment vectors $\mathbf{p}$.

Proof. Assume again that room $i$ is allocated to player $i$. If $\mathbf{p}$ is $\mathrm{EF}$, then $v_{i i}-p_{i} \geq$ $v_{i j}-p_{j}$, and $v_{j j}-p_{j} \geq v_{j i}-p_{i}$ for all $i, j \in[n]$. Since the values are within $\varepsilon$ of each other, we have that $v_{i i}-p_{i} \geq v_{j j}-p_{j}-\varepsilon$; similarly, we have that $v_{j j}-p_{j} \geq v_{i i}-p_{i}-\varepsilon$. Thus, $\left|u_{i}(i d, \mathbf{p})-u_{j}(i d, \mathbf{p})\right| \leq \varepsilon$.

Now it remains to show that values are indeed likely to be close to each other.

TheOREM 5.7. For any $\varepsilon=\varepsilon(n)=\frac{1}{o(n)}, \lim _{n \rightarrow \infty} \operatorname{Pr}\left[D^{+}(V)<\varepsilon\right]=1$.

Proof. By Lemma 5.6, it is sufficient to prove that

$$
\lim _{n \rightarrow \infty} \operatorname{Pr}\left[\forall i, j, k \in[n],\left|v_{i j}-v_{i k}\right|<\varepsilon\right]=1 .
$$

Observe that the probability that player $i$ evaluates room 1 at least at $\varepsilon$ is exactly $(1-\varepsilon)^{n-1}$. By symmetry, this is true for any room $j \in[n]$. Taking a union bound over all players and rooms, we obtain that $\operatorname{Pr}\left[\exists i, j \in[n]\right.$ s.t. $\left.v_{i j} \geq \varepsilon\right] \leq n^{2}(1-\varepsilon)^{n-1}$. However, for $\varepsilon=1 / o(n)$, we have that $\lim _{n \rightarrow \infty} n^{2}(1-\varepsilon)^{n-1}=0$.

\subsection{The Benefit Is Significant in Practice}

Above we analytically established the potential for significantly reducing disparity by using the maximin solution. In the remainder of the section we demonstrate the practical benefit of the maximin solution with respect to real-world instances that were submitted by Spliddit users.

In our empirical results, we compare the maximin solution to an arbitrary EF solution, which is obtained by solving a feasibility linear program without an optimization objective. In contrast, the theoretical analysis compares the maximin solution to the worst EF solution. We note that similar empirical results are obtained when comparing the maximin solution to the algorithm of Abdulkadiroğlu et al. [2004].

The comparison is in terms of both of our main objectives, $D$ and $U$ (which are simultaneously optimized by the maximin solution). We expected that $D$ would be significantly lower, and $U$ significantly higher, in the maximin solution compared to an arbitrary EF solution.

We focus our analysis on 1,358 rent division instances involving 3,682 players, which were submitted on Spliddit between January 2015 and December 2015. The number of instances for each number of players $2,3,4,5,6,7,8,9$ is $698,445,160,35,9,8,1$, 


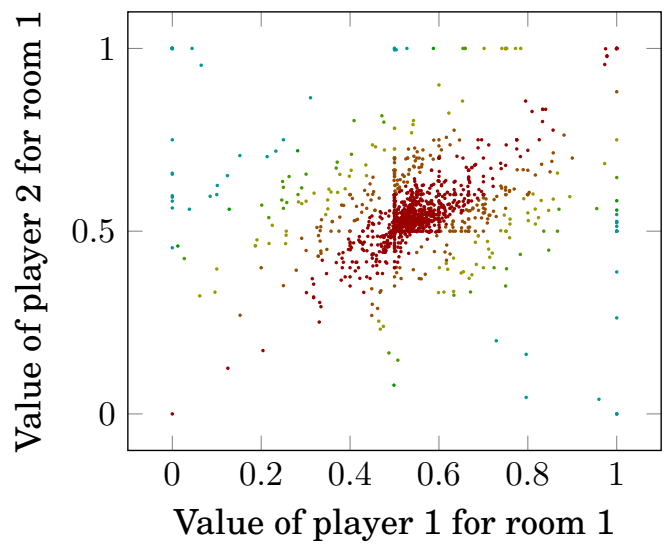

Fig. 1: The distribution of values for two player Spliddit instances (normalized to a total rent of $\$ 1$ ).

2 , respectively. We only use instances that include two, three or four players, for which we have at least 160 instances in the database and for which obtaining statistical significance was possible. Importantly, note that this is a small subset of the 13,277 rent division instances created by Spliddit users; this is because we selected instances very conservatively, to ensure the ecological validity of our analysis. For example, Spliddit allows a "live demo" mode of interaction, and we excluded instances created that way.

To illustrate users' values for rooms in the Spliddit dataset, we present Figure 1, which visualizes the distribution for 2-player instances. The $\mathrm{x}$ axis shows the value of player 1 for room 1, and the y axis shows the value of player 2 for room 1 . The total rent is normalized to $\$ 1$, so each player's value for room 2 is simply the complement of the displayed value; that is, the point $(x, y)$ corresponds to an instance where the values of player 1 are $(x, 1-x)$, and those of player 2 are $(y, 1-y)$. The diagonal from points $(0,0)$ to $(1,1)$ represents the points in which players completely agree on the rooms' values. We color each instance according to its distance from this line, using shades of red for shorter distances, and shades of blue for longer distances.

The figure reveals several interesting phenomena. First, there is a significant cluster of instances which is centered on or close to the $(0.5,0.5)$ mark, implying that both players are indifferent between the two rooms. Second, we see a "cross" centered at the $(0.5,0.5)$ point, in which one of the players is indifferent, while the other player prefers one of the two rooms. Third, there are some instances in which one or both of the players are obstinate (i.e., $x \in\{0,1\}$ or $y \in\{0,1\}$ ), that is, they desire a specific room at any cost.

Let us now turn to the comparison we promised above. Given a rent division instance $V$, let $\mathbf{p}^{*}$ denote the price vector associated with the maximin solution, and $\mathbf{p}^{E F}$ denote the price vector associated with an arbitrary EF solution, as discussed earlier. As before, we let $D(\mathbf{p})$ and $U(\mathbf{p})$ denote the social disparity and utility of the worst-off player under price vector $\mathbf{p}$ (assuming a welfare-maximizing assignment of players to rooms). The improvement in social disparity $D$ from using the maximin price vector over the EF vector is defined as $D\left(\mathbf{p}^{E F}\right)-D\left(\mathbf{p}^{*}\right)$, and the improvement in the utility of the worst-off player $U$ from using the maximin price vector over the EF vector is defined as $U\left(\mathbf{p}^{*}\right)-U\left(\mathbf{p}^{E F}\right)$.

Figure 2 shows the percentage of improvement out of the total rent in $D$ and $U$. As shown by the figure, for $n=2,3,4$, the disparity associated with the maximin outcome 


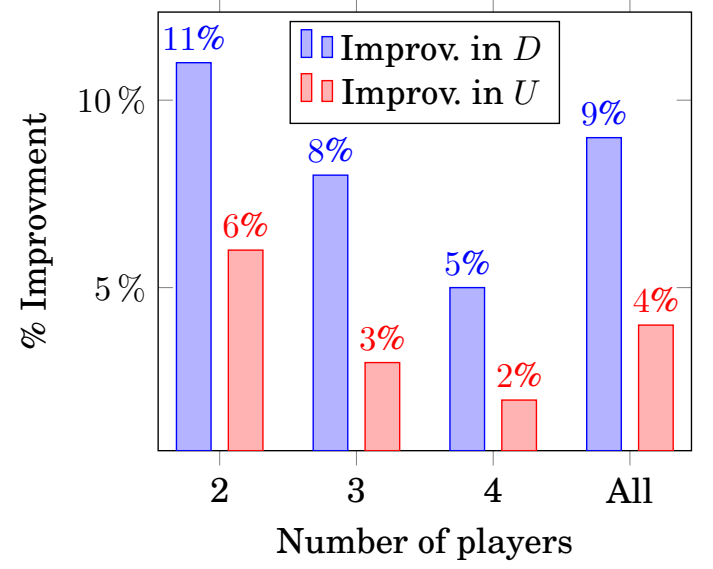

Fig. 2: Average percentage of improvement (out of the total rent) in social disparity $D$ and utility of the worst-off player $U$ when using the price vector associated with the maximin solution, compared to an arbitrary EF solution, on Spliddit instances.

is significantly lower than that of the $\mathrm{EF}$ outcome (9\% of the total rent on average), and the utility of the worst-off player associated with the maximin outcome is significantly higher than that of the EF outcome (4\% of the total rent on average). This trend is exhibited with respect to each value of $n$.

We note the following points. First, the degree of improvement in both $D$ and $U$ becomes smaller as the number of players grows, which is in the same spirit as the results of Section 5.1. However, even in cases where the improvement is relatively small, it still makes a qualitative difference, for example, when the maximin solution achieves zero disparity, and the arbitrary EF solution achieves strictly positive disparity (we discuss this fact in the next section). In addition, as noted above, the vast majority of Spliddit instances include two or three players, for which the improvement in $D$ and $U$ is higher than four players. Lastly, although this is not shown in the figure, an improvement in both $D$ and $U$ occurs in over $90 \%$ of the instances, for $n \in\{2,3,4\}$.

\section{USER STUDY}

In the previous sections, we established, both theoretically and empirically, the benefits of the maximin approach to computing envy-free solutions for rent division problems. The question addressed by this section is, are people willing to accept such solutions in practice? To answer this question, we conducted the following user study.

\subsection{Study Design}

People who used the Spliddit service during the year 2015 were invited (via email) to participate in a short study to evaluate the new allocation method. We targeted users who participated in rent division instances on Spliddit that included 2, 3 or 4 players. In order to use Spliddit one need not supply an email address; users can opt to send out URLs to other users, which is what the vast majority of users choose to do. We only contacted users who supplied their email address - a relatively small subset of the users who were involved in rent division instances.

All participants were given a $\$ 10$ compensation that did not depend on their responses. In total, the invitation email was sent to 344 Spliddit users, of which 46 users (13\%) chose to participate. The study was approved by the Institutional Review Board (IRB) of Carnegie Mellon University. 


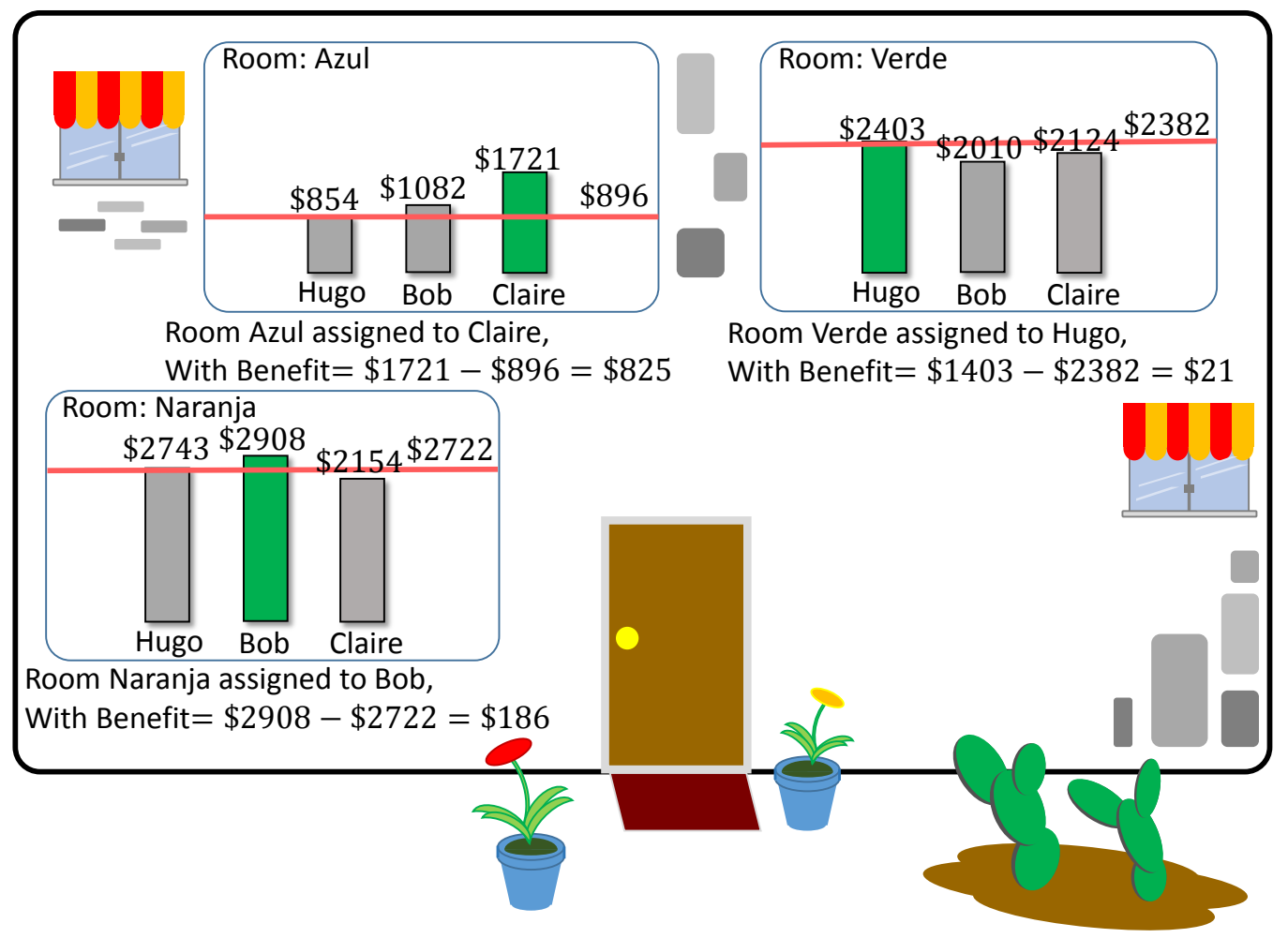

Fig. 3: A visualization of a problem instance in the user study (from the point of view of a tenant named Hugo).

The study followed a within-subject design, by which each of the subjects was shown, in random order, an arbitrary EF solution (as discussed in Section 5.2) and the maximin solution, applied to their original problem instance.

Importantly, we wished to preserve the privacy of players regarding their evaluations over the different rooms. Therefore, each player that participated in the study was shown a slightly modified version of their own rent division problem. Information that was already known to each subject was identical to the original Spliddit instance, including the total rent, the number of rooms, their names, the subject's own values for the different rooms, and the allocation of the rooms to the players. Information that was perturbed to preserve the privacy of the other players included their names, which were changed to "Alice", "Bob" or "Claire", depending on whether there were 2, 3, or 4 players; and the other players' valuations, which were randomly increased or decreased by a value of up to $15 \%$ under the constraint that the total rent is unchanged, and that player valuations are still valid (non-negative and sum to the total rent).

Figure 3 shows an example of the arbitrary EF allocation for one of the instances in the study, from the perspective of a player called Hugo. The allocation of Hugo (room Verde, utility=\$21) is shown in the "window" at the right-hand side of the "house". The value of this room for each of the players is displayed using a bar graph, with Hugo's own value highlighted via the green bar. The price paid by Hugo for room Verde (\$2382) is visualized as a horizontal line "cutting" through the value bars of the players. This provides a vivid graphical description of the values and utilities of the players for this 


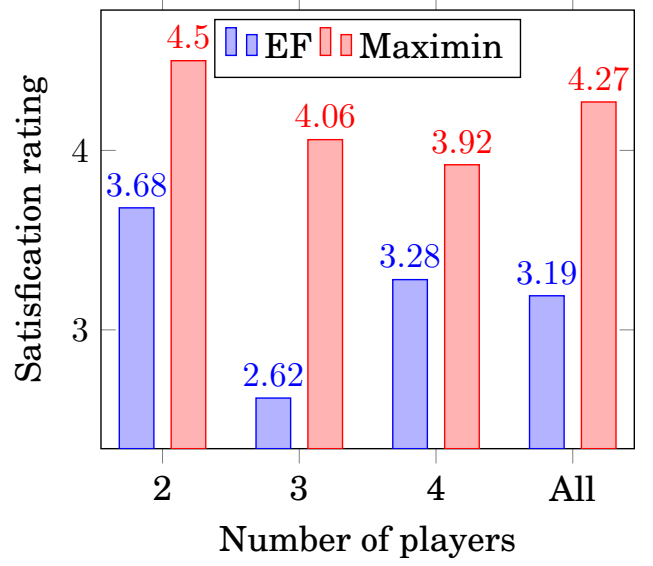

(a) Individual.

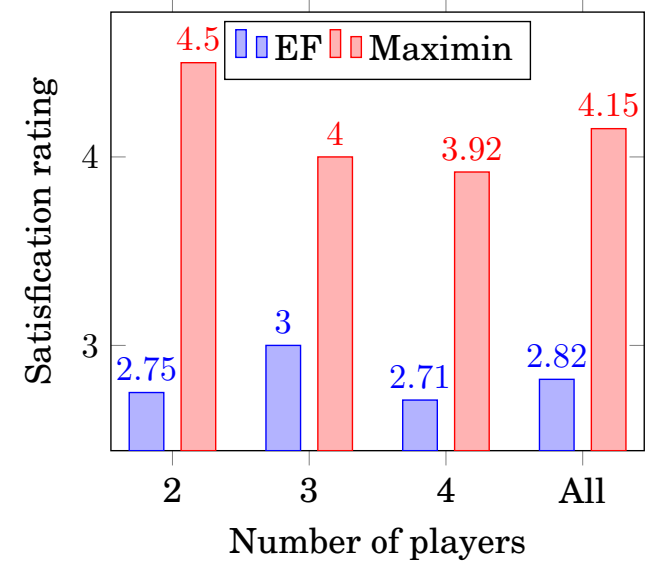

(b) Others.

Fig. 4: Results of the user study.

room, and makes it easy for participants to reason about fairness properties relating to the proposed solution. For example, it is easy to see that none of the other players envies room Verde for the proposed price. The other windows in the house show the allocations of the players Alice and Bob in a similar way.

The subjects were shown the two solutions - maximin and arbitrary EF - for the instance presented to them. Both solutions include the same room allocation, but possibly differ in the prices paid by the players. The two solution outcomes were shown in sequence, and in random order. For example, the maximin solution for the rent division instance shown in Figure 3 provides the same room assignment as the EF outcome, but the utility of all players is $\$ 344$ (compared to utilities of $\$ 21$ for Hugo, $\$ 825$ for Claire, and $\$ 186$ for Alice under the arbitrary EF outcome). Note that the disparity under the maximin solution is zero in this example, which was also the case in many of the other instances included in the study (see below).

The subjects were asked to rate two different aspects of each of the two solutions on a scale from 1 to 5 , with 1 being least satisfied and 5 being most satisfied. The two aspects are the subject's individual allocation, and the allocations of the other players. The two questions were phrased as follows (using the rent division instance of Figure 3 for illustration purposes):

(Individual). "This question relates to your own allocation. In other words, we would like you to pay attention only to your own benefit. How happy are you with getting the room called Verde for $\$ 2,382$ ?"

(Others). "This question relates to the allocation for everyone else. How fair do you rate the allocation for Bob and Claire?"

In both questions, players were able to write an argument or justification for their rating. To cancel order effects, the two questions were presented in random order.

\subsection{Results}

We hypothesized that players would rate their own allocation under the maximin solution significantly higher than under the EF outcome, and similarly for the allocation of the other participants. Figure 4 shows the results of the user study. For each number of players $(2,3,4)$ we show the average satisfaction level reported for the arbitrary EF 


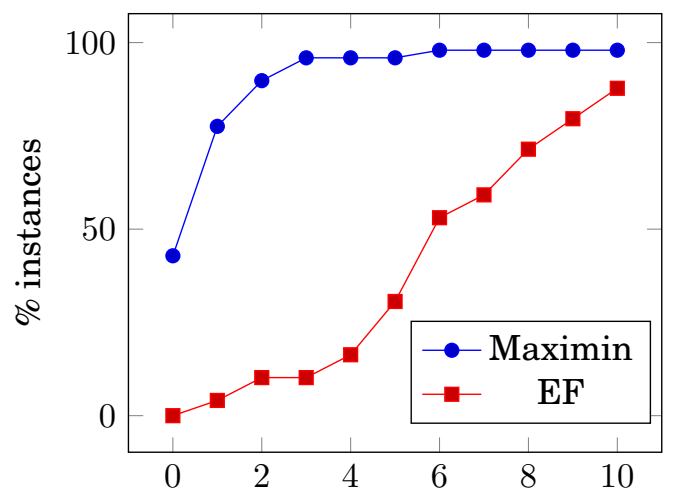

Disparity as percent of the total rent

Fig. 5: Cumulative distribution over the social disparity across all instances that were included in the user study. The $\mathrm{x}$ axis indicates the percentage of social disparity out of the total rent price.

solution and maximin solution when relating to each player's individual outcome (left chart), and others' outcomes (right chart). In all cases, the maximin solution is rated significantly higher than the envy-free solution for both questions, passing a Wilcoxon signed-rank test with $p<0.04$.

Anecdotally, based on textual feedback, subjects had a good understanding of the experiment. As an example, on the instance of Figure 3, the subject identified as Hugo wrote regarding his own outcome: "It looks like I am overpaying." And for the allocation of the other players: "They both get much more benefit."

Why did players overwhelmingly prefer the prices from the maximin solution over the arbitrary EF solution? Given the high importance attributed to social disparity when reasoning about fair division [Herreiner and Puppe 2009], we hypothesized that the price vectors of the maximin solution exhibited significantly lower disparity than the price vectors of the EF solution. This was supported by many of the textual comments relating to social disparity. Figure 5 shows the cumulative distribution of disparity across all instances that were included in the user study. The $\mathrm{x}$ axis indicates the disparity as percentage of the total rent. As shown by the figure, the disparity associated with the maximin solution is indeed significantly lower. In fact, in many instances the disparity is zero under the maximin solution. (For the $n=2$ case, Lemma 5.1 shows that the minimum disparity is zero for any instance.) We believe that this large difference in disparity played a key role in subjects' preference for the maximin solution, trumping the relatively small improvement in utilities.

\section{DISCUSSION}

The reader may wonder why we have not addressed game-theoretic issues. One reason is that envy freeness is inherently incompatible with incentive compatibility. This follows from the classic result of Green and Laffont [1979] and the fact that envy freeness implies Pareto efficiency in our setting. More importantly, we believe that, in rent division, strategic behavior does not play a significant role in practice. In particular, on Spliddit each user enters values for rooms separately, without seeing the information submitted by other users. Moreover, most users do not know how the algorithm works (we do not attempt to explain the algorithm itself, only its fairness guarantees). 
Taking a broader viewpoint, we believe that computational fair division is a prime example of how the interaction between computer science and economics can lead to novel applications. We find it particularly exciting that fundamental theoretical questions in this field have direct real-world implications [Aleksandrov et al. 2015; Kurokawa et al. 2015; Procaccia and Wang 2014]. The current paper takes the computational fair division agenda a step further, by tying together theory, experiments on real data, a carefully designed user study, and a deployed application.

\section{ACKNOWLEDGMENTS}

This work was supported by EU FP7 FET project, grant agreement n.600854; by the National Science Foundation under grants IIS-1350598, CCF-1215883, and CCF-1525932; and by a Sloan Research Fellowship.

\section{REFERENCES}

A. Abdulkadiroğlu, T. Sönmez, and M. U. Ünver. 2004. Room assignment-rent division: A market approach. Social Choice and Welfare 22, 3 (2004), 515-538.

M. Aleksandrov, H. Aziz, S. Gaspers, and T. Walsh. 2015. Online fair division: Analysing a food bank problem. In Proceedings of the 24th International Joint Conference on Artificial Intelligence (IJCAI). 2540-2546.

A. Alkan, G. Demange, and D. Gale. 1991. Fair allocation of indivisible goods and criteria of justice. Econometrica 59, 4 (1991), 1023-1039.

E. Aragones. 1995. A derivation of the money Rawlsian solution. Social Choice and Welfare 12 (1995), 267-276.

S. J. Brams and D. M. Kilgour. 2001. Competitive fair division. Journal of Political Economy 109 (2001), 418-443.

N. Dupuis-Roy and F. Gosselin. 2011. The simpler, the better: A new challenge for fair-division theory. In Proceedings of the 33rd Annual Meeting of the Cognitive Science Society (CogSci). 3229-3234.

D. Foley. 1967. Resource allocation and the public sector. Yale Economics Essays 7 (1967), 45-98.

J. Goldman and A. D. Procaccia. 2014. Spliddit: Unleashing fair division algorithms. SIGecom Exchanges 13, 2 (2014), 41-46.

J. R. Green and J.-J. Laffont. 1979. Incentives in Public Decision Making. North Holland.

C.-J. Haake, M. G. Raith, and F. E. Su. 2002. Bidding for envy-freeness: A procedural approach to $n$-player fair-division problems. Social Choice and Welfare 19 (2002), 723-749.

D. K. Herreiner and C. D. Puppe. 2009. Envy freeness in experimental fair division problems. Theory and decision 67, 1 (2009), 65-100.

D. K. Herreiner and C. D. Puppe. 2010. Inequality aversion and efficiency with ordinal and cardinal social preferences - an experimental study. Journal of Economic Behavior \& Organization 76,2 (2010), 238-253.

F. Klijn. 2000. An algorithm for envy-free allocations in an economy with indivisible objects and money. Social Choice and Welfare 17 (2000), 201-215.

S. Kohler. 2013. Envy can promote more equal division in alternating-offer bargaining. Journal of Neuroscience, Psychology, and Economics 1, 6 (2013), 31-41.

D. Kurokawa, A. D. Procaccia, and N. Shah. 2015. Leximin allocations in the real world. In Proceedings of the 16th ACM Conference on Economics and Computation (EC). 345-362.

A. Mas-Colell, M. D. Whinston, and J. R. Green. 1995. Microeconomic Theory. Oxford University Press.

A. D. Procaccia and J. Wang. 2014. Fair enough: Guaranteeing approximate maximin shares. In Proceedings of the 14th ACM Conference on Economics and Computation (EC). 675-692.

G. Schneider and U.S. Krämer. 2004. The limitations of fair division: An experimental evaluation of three procedures. Journal of Conflict Resolution 48, 4 (2004), 506-524.

F. E. Su. 1999. Rental harmony: Sperner's lemma in fair division. American Mathematical Monthly 106, 10 (1999), 930-942.

L.-G. Svensson. 1983. Large indivisibles: An analysis with respect to price equilibrium and fairness. Econometrica 51, 4 (1983), 939-954. 